\title{
Cell lineage specification at single cell resolution
}

\author{
Jian Yang, Pentao Liu \\ Wellcome Trust Sanger Institute, Hinxton, Cambridge CB10 1HH, UK \\ Correspondence to: Jian Yang, PhD. Wellcome Trust Sanger Institute, Hinxton, Cambridge CB10 1HH, UK. Email: jy4@sanger.ac.uk. \\ Comment on: Mohammed H, Hernando-Herraez I, Savino A, et al. Single-Cell Landscape of Transcriptional Heterogeneity and Cell Fate Decisions \\ during Mouse Early Gastrulation. Cell Rep 2017;20:1215-28.
}

Received: 01 September 2017; Accepted: 03 September 2017; Published: 19 September 2017.

doi: $10.21037 /$ sci.2017.09.03

View this article at: http://dx.doi.org/10.21037/sci.2017.09.03

\section{Introduction}

Single cell technology has been widely used in developmental and stem cell biology. In mouse, single cell transcriptome has revealed the cell lineage specification in preimplantation and post-implantation stages embryo. Now Mohammed et al. investigated the dynamic cell fate commitment during the transition from peri-implantation to early post-implantation stage with single cell RNA sequencing. Except confirmation of some previous findings, the time window and cell subclusters of cell specification are more precisely determined, along with possible new mechanism for $\mathrm{X}$ chromosome re-activation and inactivation in female embryo and for exit from pluripotency and lineage commitment. These data will not only fill the missing link in mouse embryo development, but provide insights into embryo development of other mammalian species including human.

How a zygote generates a multicellular organism is one of the fundamental questions in developmental and stem cell biology. Take mouse embryo development as an example. Until 8-cell stage, each blastomere is totipotency, i.e., it can generate the first three lineages, trophoectoderm (TE), epiblast (EPI) and primitive endoderm (PE); though recent reports showed a single 4 cell blastomere's contribution to TE/ICM is biased and single cell assay also revealed heterogeneity in gene expression. At morula stage (16-32 cells), the first two lineages, TE and ICM are segregated, then at blastocyst stage, ICM specified into EPI and PE. After implantation, TE contributes to trophoblasts in placenta; EPI produces all fetal cells including germ cells and PE mostly forms extra-embryonic yolk sac (1).

In order to trace the cell specification during development, various methods and techniques have been developed and applied, including cell labelling, transplantation of cells and tissues, introduction of genetic markers by transfection or viral transduction and cell marking by genetic recombination etc. All these methods have pros and cons though (2). More recently, with the fast advance of single cell technology, the fate of cells can be monitored more precisely and in more details (3). Early this year, Posfai et al. using single cell RNA sequencing found drastically increased diversity among cells between later 16 cells stage and early 32 cells stage, suggesting the emergence of TE and ICM lineages at these stages (4). Before, Peng et al. constructed a spatial transcriptome of late mid-streak embryo [around embryo day (E) 7.0] using high resolution RNA sequencing (20 cells from four positions in each section) and correlated with regionalization of cell fates in the embryo. From these datasets, the authors created zip code mapping. To test the utility of the map, they sequenced 70 single cells, after inputting the data, the position of single cell in embryo was mapped. It turned out that 66 of 70 single cells can be mapped back to the original half-EPI (5).

There is a gap between these two reports which is the transition of embryo from peri-implantation to postimplantation. During these stages, ICM segregates into EPI and PE, EPI subsequently undergo expansion to form primitive streak to initiate gastrulation. This is also critical time window for stem cell biology as naive embryonic stem cells (ESCs) can be derived from ICM in blastocyst, whereas primed epiblast stem cells (EpiSCs) were derived from postimplantation EPI and the transcriptional and epigenetic changes during the exit from naive pluripotency is still not clear (6). To open the black box, now Mohammed et al. performed single cell RNA sequencing of ICM of E3.5, EPI and PE at E4.5, E5.5 and E6.5. E3.5 cells expressed high level of pluripotency genes; at E4.5, EPI and PE was 
clearly separated; at E5.5, naive pluripotency gene Nanog was down-regulated accompanied by increased primed pluripotency maker Pou3f1; at E6.5, the embryo cluster into four groups, visceral endoderm (from PE), a primitive streak population and two subclusters of non-committed EPI cells (7).

In female mouse embryo, between E3.5 and E4.5, the paternal $\mathrm{X}$ chromosome is reactivated, then from E5.5 on, one $\mathrm{X}$ chromosome is randomly inactivated (8). Consistent with the in vivo phenomenon, female naive ESCs also have two activated $\mathrm{X}$ chromosomes whereas one $\mathrm{X}$ chromosome is inactivated in female EpiSCs (6). The new single cell transcriptome data confirmed the previous observation, moreover, they found strong association between $\mathrm{X}$ chromosome reactivation and Pousf1 and one of its interacting partner $Z f b \times 3$ at E3.5; while increased expression of Dnmt $3 a$ and $Z f p 57$ is associated with the following $\mathrm{X}$ chromosome inactivation, which will provide further insights into the mechanism of $\mathrm{X}$ chromosome reactivation and inactivation (7).

Prior to the segregation of EPI and PE at E4.5, specific EPI and endoderm genes are co-expressed at E3.5. The authors' data clearly showed genes enriched for EPI and PE respectively at E4.5; at E3.5, though subsets of EPI and PE genes were expressed, no lineage subgroups were observed. Besides identifying genes such as $F g f 4$ and $F g f r 2$ known to derive EPI and PE specification, they also found Pdgfra, Top2b, Sox 17, Gata4 and Pdk2 were associated with PE fate, and Morc1, Nanog, Dppas and Pdpn were associated with EPI fate (7).

From E5.5 to E6.5, the cells begin to exit pluripotency and undergo lineage commitment. The data revealed that at E5.5, though some genes associated with anteriorposterior polarity and the primitive streak, no apparent substructure or expression of primitive streak markers were expressed, whereas at E6.5, four subgroups were identified as mentioned above, in EPI, the expression of $O t x 2$, a gene associated with exit from pluripotency increased, along with several polycomb genes, which suggests the importance of polycomb complex in establishing transcriptional control in the non-committed EPI cells (7).

Finally, they investigated the transcriptional noise at different embryo stages, which showed high noise at E3.5 unspecified cells, then gradually reduced and at E6.5, the primitive streak has much lower noise than the uncommitted EPI. These data suggest that there is possibility of increased transcriptional noise before lineage commitment as also observed in other systems $(7,9)$.
In summary, this study provides us more information about the dynamic changes during the transition from peri-implantation to early post-implantation stages, which mark the lineage segregation, exit from pluripotency and differentiation. However, the commitment of TE is missing in this report. Considering trophoblast stem cells can be derived from either E3.5 blastocyst or E6.5 extra-embryonic ectoderm (10). It'll be interesting to investigate the fate of TE during this transition stage at single cell level.

The new study may also shed light on the embryo development of human and other mammalian species. Several reports investigated transcriptome of human preimplantation embryos by single cell sequencing, from which not only a number of genes with conserved expression as in mouse was observed, but also significant difference between human and mouse was identified $(11,12)$. Moreover, $\mathrm{X}$ chromosome re-activation and inactivation in female human blastocyst is different as well (13). Because of the ethical issues, it's almost impossible to study the early human post-implantation embryos. However, recently developed in vitro culture systems with which human blastocyst can self-organize into post-implantation embryo like structure may facilitate the study of human post-implantation embryo development $(14,15)$.

In all, the advanced single cell technology will provide a powerful tool for developmental and stem cell scientists to map the cell fates from a zygote to the whole organism which can be translated into clinical application.

\section{Acknowledgements}

None.

\section{Footnote}

Conflicts of Interest: The authors have no conflicts of interest to declare.

\section{References}

1. Chazaud C, Yamanaka Y. Lineage specification in the mouse preimplantation embryo. Development 2016;143:1063-74.

2. Kretzschmar K, Watt FM. Lineage tracing. Cell 2012;148:33-45.

3. Wen L, Tang F. Single-cell sequencing in stem cell biology. Genome Biol 2016;17:71.

4. Posfai E, Petropoulos S, de Barros FR, et al. Position- 
and Hippo signaling-dependent plasticity during lineage segregation in the early mouse embryo. Elife 2017;6. pii: e22906.

5. Peng G, Suo S, Chen J, et al. Spatial Transcriptome for the Molecular Annotation of Lineage Fates and Cell Identity in Mid-gastrula Mouse Embryo. Dev Cell 2016;36:681-97.

6. Nichols J, Smith A. Naive and primed pluripotent states. Cell Stem Cell 2009;4:487-92.

7. Mohammed H, Hernando-Herraez I, Savino A, et al. Single-Cell Landscape of Transcriptional Heterogeneity and Cell Fate Decisions during Mouse Early Gastrulation. Cell Rep 2017;20:1215-28.

8. Lessing D, Anguera MC, Lee JT. X chromosome inactivation and epigenetic responses to cellular reprogramming. Annu Rev Genomics Hum Genet 2013;14:85-110.

9. Richard A, Boullu L, Herbach U, et al. Single-Cell-Based Analysis Highlights a Surge in Cell-to-Cell Molecular Variability Preceding Irreversible Commitment in a Differentiation Process. PLoS Biol 2016;14:e1002585.

doi: $10.21037 /$ sci.2017.09.03

Cite this article as: Yang J, Liu P. Cell lineage specification at single cell resolution. Stem Cell Investig 2017;4:76.
10. Latos PA, Hemberger M. From the stem of the placental tree: trophoblast stem cells and their progeny. Development 2016;143:3650-60.

11. Blakeley P, Fogarty NM, del Valle I, et al. Defining the three cell lineages of the human blastocyst by single-cell RNA-seq. Development 2015;142:3151-65.

12. Durruthy-Durruthy J, Wossidlo M, Pai S, et al. Spatiotemporal Reconstruction of the Human Blastocyst by Single-Cell Gene-Expression Analysis Informs Induction of Naive Pluripotency. Dev Cell 2016;38:100-15.

13. Petropoulos S, Edsgard D, Reinius B, et al. Single-Cell RNA-Seq Reveals Lineage and X Chromosome Dynamics in Human Preimplantation Embryos. Cell 2016;165:1012-26.

14. Deglincerti A, Croft GF, Pietila LN, et al. Selforganization of the in vitro attached human embryo. Nature 2016;533:251-4.

15. Shahbazi MN, Jedrusik A, Vuoristo S, et al. Selforganization of the human embryo in the absence of maternal tissues. Nat Cell Biol 2016;18:700-8. 